\title{
Nanoestructuras y su caracterización por medio de microscopía electrónica de transmisión; ciencia y arte ${ }^{\diamond}$
}

\section{Nanostructures and their characterization by transmission electron microscopy; science and art}

\author{
Miriam-Carolina Mendoza Ramírez,* Miguel Avalos Borja*,**
}

\begin{abstract}
The description of nanometric systems is still being a challenging topic, for this reason the transmission electron microscopy (TEM) scope is exemplified in a didactic way using several nanosystems (Au nanoparticles and thin Si films). Throughout this work, conventional TEM techniques such as bright field (BF), dark field (DF), high angle annular dark field (HAADF), selected area electron diffraction (SAED) and electron energy loss spectroscopy (EELS) are shown, emphaticizing the differences with less conventional techniques such as convergent beam electron diffraction (CBED), large angle convergent beam electron diffraction (LACBED) and precession electron diffraction (PED). Also, some practical suggestions are given for describing contrast found on several TEM techniques, offering a striking, clear and didactic vision of the current scopes of TEM in Mexico.
\end{abstract}

KEYWORDS: transmission electron microscopy, electron diffraction, precession and convergent beam.

RESUMEN: En el presente trabajo se describen de forma didáctica los alcances de la microscopía electrónica de transmisión (TEM) para el estudio de nanosistemas. Las ilustraciones mostradas se adquirieron empleando muestras ilustrativas (nanopartículas de Au y películas de Si). A lo largo de este escrito se ejemplifica el uso de algunas técnicas TEM convencionales, como BF, DF, HAADF, SAED y EELS, así como también se ejemplifica el empleo de técnicas avanzadas de difracción, como CBED, LACBED y PED. También se brindan algunas sugerencias prácticas que permitirán describir y diferenciar de forma sencilla el contraste observado en algunas de las técnicas disponibles en el TEM. Todo con el objetivo de ofrecer una visión, llamativa, clara y didáctica de los alcances actuales de la microscopía electrónica en México.

PALABRAS CLAVE: microscopía electrónica de transmisión, difracción de electrones, precesión y haz convergente.

Recibido: 7 de febrero de 2020.

Aceptado: 4 de mayo de 2020.

${ }^{\diamond}$ Los autores agradecen al LINAN y a la UNaC, por facilitar el uso de sus instalaciones y equipos para ilustrar el presente trabajo, también a los técnicos académicos Héctor Gabriel Silva Pereyra e Israel Gradilla Martínez, así como a Óscar Edel Contreras López, por las facilidades brindadas en apoyo al presente trabajo.

* Instituto Potosino de Investigación Científica y Tecnológica, División de Materiales Avanzados, San Luis Potosí, S.L.P., México.

**Autor de correspondencia: miguel.avalos@ipicyt.edu.mx 


\section{Introducción}

Los materiales nanoestructurados han sido señalados como candidatos ideales para el desarrollo de aplicaciones novedosas en áreas diversas (Zhang et al., 2005; Daniel et al., 2004; Tomar et al., 2013; Das et al., 2011). Incluyendo catálisis (Zhou et al., 2012), ciencia de materiales (Morin et al., 2011) o medicina (Eustis y El-Sayed, 2006). De manera particular, las nanopartículas anisotrópicas han atraído gran atención debido a sus propiedades ópticas y a su inusual comportamiento físico y químico (Vigderman et al., 2012; Major et al., 2013). Estas nanopartículas se caracterizan por crecer con morfologías no definidas por el estado de equilibrio (bastones, platos, decaedros, etc.) (Barmparis et al. 2015) las cuales comúnmente se preparan usando métodos coloidales (Millstone et al., 2008; Nikoobakht y El-Sayed, 2003; Yao et al., 2017). El control y entendimiento de los sistemas nanométricos coloidales es fundamental para el desarrollo de aplicaciones, lo cual ha incentivado la creación de distintas técnicas capaces de acercarnos al "nanomundo". Estas, en su conjunto, se conocen como "técnicas de caracterización". A pesar de que la comprensión de cada una de estas técnicas requiere cierta profundidad, de manera muy general pueden dividirse en aquellas que nos permiten obtener: espectros, imágenes o patrones de difracción.

Las técnicas espectroscópicas, como UV-VIS NIR, RAMAN, EDS, entre otras, permiten caracterizar propiedades como la respuesta óptica y la composición química. Por otra parte, las técnicas que producen imágenes, como SEM, BF TEM o CL, son capaces de diferenciar morfología, composición e incluso propiedades como luminiscencia. Las limitaciones y requerimientos para cada una de estas técnicas están relacionadas generalmente con el tipo de radiación que se emplea como sonda ya que es posible hacer incidir: fotones, electrones o iones de distintos materiales y con distinta energía.

Finalmente, para el análisis de estructura de materiales, la técnica por excelencia es la difracción, este fenómeno es observado siempre que exista una fuente de radiación monocromática con longitud de onda comparable con la distancia interatómica de un cristal, actualmente ya se han desarrollado sistemas que usan rayos $\mathrm{X}$, electrones o neutrones.

El presente trabajo se centra en una técnica imprescindible para el estudio del mundo nanométrico: la microscopía electrónica de transmisión (TEM). Esta versátil y poderosa técnica es capaz de adquirir: imágenes (en campo claro u oscuro, en barrido, con contraste de número atómico, con contraste de difracción tanto de amplitud como de fase), espectros (caracterizando composición química, espesor, estados de oxidación) o patrones de difracción (observando la estructura de un material simultáneamente, describiendo celdas unitarias, distancias interplanares, ausencias sistemáticas, grupo espacial, entre otros) mediante el uso de sus diversas variantes (Zou et al., 2012).

Las muestras para TEM se fabrican típicamente con espesores menores a $100 \mathrm{~nm}$, las cuales deben poseer cierta tolerancia al daño por radiación io- 
nizante causado por electrones o en su defecto ser sometidas a preparación especial usando metales pesados y la técnica de corte por ultramicrotomo (esto último es especialmente válido para muestras biológicas).

A lo largo de este trabajo, se describen de forma didáctica: las técnicas típicas para el estudio de materiales nanométricos y la obtención de información química, morfológica y estructural para diversos sistemas nanométricos anisotrópicos; incluyendo platos, bastones y decaedros, y se introduce la aplicación de técnicas avanzadas de difracción, como haz convergente (CBED) (Morniroli, 2006) y haz convergente de amplio ángulo (LACBED) (Tanaka et al., 1980; Morniroli, 2003.), también se presenta una técnica única en el país: la técnica de difracción de electrones en precesión (PED) (Avilov et al., 2007). Para ilustrar las técnicas de difracción en TEM, se emplearon muestras didácticas de Si. Este trabajo brinda algunas recomendaciones prácticas que permitirán discernir fácilmente el significado del contraste en las micrografías TEM que observamos día con día.

\section{Experimental}

\section{Sintesis de nanopartículas de $\mathrm{Au}$}

Para ilustrar el presente trabajo se sintetizaron nanopartículas de oro de tres morfologías distintas, nanorrodillos, nanoplatos, y nanodecaedros. Todas las partículas se sintetizaron usando procesos de nucleación heterogénea, mediados por semillas, usando surfactantes y medios distintos. Para sintetizar decaedros y barras, se empleó el método de preparación de semillas de Turquevich (Nikoobakht y El-Sayed, 2003; Sánchez-Iglesias et al., 2006), mientras que para la preparación de platos se empleó un proceso de tipo Polyol (Kan et al., 2010).

\section{Preparación de muestras}

\section{Usando desbaste iónico}

Para la fabricación de películas delgadas de Si se empleó la técnica de desbaste iónico, dicha técnica permite adelgazar una pieza de material sólido de dimensiones mesométricas hasta convertirlo en una lámina delgada de espesor nanométrico. Típicamente el cristal mesométrico es adelgazado hasta formar un agujero en el centro de la muestra, cuyos bordes acuñados tienen espesores nanométricos, siendo esta la única parte observable en TEM. Para la preparación de muestra TEM, se seleccionó un monocristal de Si de aproximadamente de $1 \mathrm{~mm}$ de espesor, el cristal se adhirió a una rejilla ranurada usando pegamento epóxico. Más adelante, la muestra fue sometida a un proceso sistemático de adelgazamiento usando: discos de SiC (hasta100 $\mu \mathrm{m}$ de espesor aprox.), un desbastador tipo trípode Polisher 590W SPI (hasta10 $\mu \mathrm{m}$ de espesor aprox.), y, finalmente, un erosionador iónico Precision Ion Polishing System de Gatan. 


\section{Usando nanofabricación}

Puesto que la técnica de desbaste iónico presenta distintas limitaciones técnicas, existe una alternativa usando sistemas de nanofabricación acoplados a un microscopio de barrido, mediante este sistema es posible fabricar muestras especializadas que son conocidas como "lamelas". Estas muestras son segmentos rectangulares con variaciones constantes de espesor, en cuyo borde se puede apreciar una cuña de unos pocos nanómetros de espesor.

El proceso de preparación de lamela para una muestra de $\mathrm{Si}$, se ilustra en la figura 7. Para las ilustraciones mostradas en el presente artículo, se empleó un equipo de nanofabricación acoplado a un microscopio electrónico de barrido JEOL JIB-4500, usando cañones de Pt y Ga, así como el uso de un nano-manipulador Omniprobe.

\section{Caracterizaciones usando microscopía electrónica de transmisión (TEM)}

Todas las caracterizaciones TEM fueron realizadas usando un microscopio FEI Tecnai-F30 TEM a 300 kV. Para analizar la morfología se adquirieron imágenes en campo claro (BF) y oscuro (DF). Para los experimentos de difracción se usaron cuatro técnicas distintas: difracción de área selecta (SAED), difracción en precesión de haz (PED), difracción con haz convergente (CBED) y difracción de haz convergente de amplio ángulo (LACBED). Se hizo uso de bayonetas de inclinación sencilla y de doble inclinación. Las micrografías se adquirieron usando una cámara digital Gatan ${ }^{\mathrm{TM}}$ Orius SC200. En las micrografías mostradas, el contraste, brillo y gamma, fueron modificados ligeramente para maximizar el contraste de visualización.

\section{Espectroscopía EELS}

La espectroscopía de pérdida de energía de electrones (EELS), permite observar la interacción inelástica de los electrones al atravesar una muestra. El presente texto no profundiza en el fundamento y la realización práctica de esta técnica; sin embargo, la consulta del libro de R. F. Egerton se recomienda ampliamente para este propósito (Egerton, 1986).

De forma breve, para la obtención de un espectro EELS, el haz transmitido es recolectado por un prisma magnético, el cual deflecta los electrones en función de la pérdida de energía que estos mismos experimentan al atravesar la muestra. Esta poderosa técnica tiene distintas variantes y permite analizar (en distintas partes del espectro y en función de la monocromaticidad del haz) propiedades como: espesor, composición química, estados de oxidación y de valencia, por mencionar algunos.

En este trabajo, se muestra un ejemplo didáctico para el cálculo de espesor de una muestra plana de Au usando espectroscopía EELS y el software Digital Micrograph (DM) por medio de la metodología "Log-ratio". Mediante esta metodología, es posible estimar el espesor relativo de una muestra 
como función del camino libre inelástico medio (MFP), simplemente adquiriendo espectros EELS en la zona de bajas pérdidas (de 0 a 180 eV, aproximadamente) y precisando los siguientes parámetros: la energía del haz incidente $\left(\mathrm{E}_{0}\right)$, el semiángulo de convergencia $(\alpha)$, el semiángulo de recolección ( $\beta$ ) así como el número atómico efectivo (Egerton, 1986).

\section{Campo claro (BF)}

Este es el modo de operación básico en TEM, se caracteriza porque los objetos de interés contrastan en escala de gris a negro, sobre un fondo claro. Para las imágenes adquiridas en BF se usó iluminación paralela convencional y apertura objetiva (para mejorar el contraste debido a cambios en masa-espesor o difracción).

\section{Campo oscuro (DF)}

Este es un modo de operación básico, que permite resaltar el contraste generado por la difracción de un haz difractado en particular. La aplicación de esta técnica se ejemplifica en la figura 3 h. El procedimiento para obtener una imagen en campo oscuro consiste en alinear uno de los haces difractados con el eje óptico del microscopio, filtrando esta información mediante el uso de la apertura objetiva.

Adquisición de patrones de difracción de área selecta (SAED)

Esta es la técnica básica y más empleada en cuanto a difracción, aunque irónicamente es una de las menos útiles, puesto que la difracción SAED se ve fuertemente afectada por el comportamiento "dinámico" de los electrones. Motivo por el cual esta técnica no se recomienda para la determinación de modelos estructurales. Para obtener un patrón de difracción convencional SAED basta con seleccionar un área de interés por medio de la apertura de área selecta (SA) y ejercer cambios en las lentes intermedias del microscopio, dichos cambios provocan que en la pantalla fluorescente del microscopio se observe el plano focal posterior en lugar del plano imagen. Mediante un patrón de difracción SAED podemos observar algunas de las simetrías características de un cristal.

\section{Técnicas de difracción con precesión de haz (PED)}

La alineación para la obtención de patrones en precesión se muestra en la figura $6 \mathrm{~b}$. Esta técnica ofrece importantes ventajas en comparación con la adquisición convencional SAED. Para este tipo de adquisición se precisa el uso de bobinas deflectoras, las cuales se ubican en un plano superior e inferior a la muestra y tienen acción directa sobre el haz de electrones. Las bobinas deflectoras tienen la función de rotar el haz de electrones para formar un ángulo sólido en movimiento de precesión. El haz cónico y cíclico que incide y atraviesa la muestra es reintegrado en un punto por medio de un segundo grupo de bobinas deflectoras situadas en un plano inferior a la muestra. 
Mediante esta técnica se pueden obtener patrones que lucen de manera similar a un patrón SAED (cuya indexación se basa en los mismos criterios) pero con la ventaja de que mediante este tipo de adquisición, se minimizan los efectos de doble difracción y de difracción dinámica. Además, mediante el uso de esta técnica, es posible analizar de forma integral la información difractada en los "relrods" del espacio recíproco, midiendo patrones con intensidad cuantificable. Siendo la técnica ideal para el estudio estructural detallado de cristales nanométricos (Midgley y Eggeman, 2015; Vincent y Midgley, 1994). Los patrones PED mostrados en el presente trabajo fueron adquiridos usando haz paralelo y una unidad de precesión SpinningStar P-20, con ángulos de precesión menores a 2 grados.

\section{Técnicas de difracción con haz convergente (CBED y LACBED)}

La técnica LACBED es la más desafiante de las técnicas de difracción, para su realización se requiere un amplio conocimiento cristalográfico y excelentes habilidades operativas (Morniroli, 2003 y 2006), debido a que la obtención de una imagen con contraste adecuado depende de distintas variables y de varios recursos indispensables, como: un portamuestras de doble inclinación, un microscopio con aperturas condensadoras grandes y una sonda pequeña (STEM Nanoprobe), además de que se requiere un área iluminada lo suficientemente grande. Por tales razones, se precisan muestras relativamente grandes y gruesas (muestras mayores a $5 \mu^{2}$ y con espesores entre los 15 y $30 \mathrm{~nm}$ ).

Considerando los retos inherentes a la técnica, se propuso ilustrar el presente artículo con muestras de Si monocristalino, mismas que fueron preparadas por desbaste iónico en rejilla de una ranura y muestras tipo "lamela" en media rejilla. Para estas muestras fue posible adquirir, patrones de Kossel y patrones LACBED BF en distintos ejes zonales. Empleando las siguientes condiciones operativas: Modo STEM nanoprobe, con una apertura C2 de $200 \mu \mathrm{m}$. Para separar los patrones de discos superpuestos (Kossel) se aplicó la metodología de Tanaka,16 la cual consiste en separar los discos realizando movimientos de la muestra de forma paralela al eje óptico del microscopio, para nuestro caso particular, se alcanzaron condiciones de foco a valores negativos del eje óptico alrededor de las $280 \mu \mathrm{m}$. De acuerdo con esta metodología, después de separar los discos, es posible filtrar la información obtenida, seleccionando un haz con la apertura de área selecta; si el usuario selecciona el haz trasmitido se obtiene un patrón LACBED en campo claro (LACBED BF) en cambio si se selecciona un haz difractado se observa un patrón LACBED en campo oscuro.

\section{Resultados}

En esta sección se ilustran los resultados típicos obtenidos por las distintas técnicas disponibles en TEM Tecnai-F-30. En las figuras (1-5) se pueden observar los resultados obtenidos en cuanto a las síntesis de nanopartículas. Se lograron 
sintetizar éxitosamente barras (figura 2), decaedros (figuras 4a-f) y partículas tipo plato con morfologías triangulares y hexagonales (figuras 1a y 4 a-b). Las muestras fueron analizadas mediante distintas técnicas en el TEM, las micrografías y espectros que se muestran en esta sección se seleccionaron para resaltar propiedades particulares de cada una de las técnicas de TEM empleadas.

\section{Imagen y espectroscopía de pérdida de energía (EELS)}

En la figura 1a, se observa el espectro EELS representativo para la partícula triangular insertada en la gráfica de la figura 1a, en el rango correspondiente a bajas pérdidas de energía. En esta micrografía se observan dos partículas con morfología triangular las cuales tienen una diferencia de contraste significativa. Ambas partículas fueron analizadas mediante espectroscopía EELS, los espectros de ambas partículas presentaban el plasmón característico de $\mathrm{Au}$, similares al mostrado en la figura 1a, por lo cual se determinó que ambas poseen la misma composición. Dado que la composición es la misma para ambas partículas, la diferencia significativa de contraste puede ser atribuida únicamente a cambios en el espesor. Aunque este cambio no se observa de forma directa en la micrografía, es claro y evidente en los perfiles observados en la figura $1 b-c$. Estos perfiles fueron realizados usando espectroscopía EELS y la metodología "log-ratio" disponible en el software DM ${ }^{\circledR}$. Las trazas (B y C) se indican en la micrografía de la figura la y estas corresponden a los perfiles mostrados en la figura 1 (b y c), respectivamente. En estas trazas puede observarse claramente el perfil de cada una de las partículas, relacionándose con el contraste observado. Mediante este perfil se logró inferir la morfología tridimensional de las partículas, mostrada esquemáticamente en los perfiles mostrados: para (b) una partícula tetraédrica y para (c) un plato plano con bordes acuñados.

De esta manera, se puede complementar el uso de una técnica de análisis espectroscópico como EELS, con el uso de una técnica de imagen TEM BF

Figura 1. En esta figura su puede apreciar un análisis químico y de morfología realizado para las partículas mostradas en el espectro (a) usando espectroscopía EELS en la zona de bajas pérdidas. En el espectro (a) pueden apreciarse los tres picos característicos del plasmón de Au, así como la banda característica de la capa $\mathrm{O}_{\alpha, \beta}$. Los perfiles de espesor mostrados en (b y c) permitieron establecer diferencias morfológicas entre las partículas.
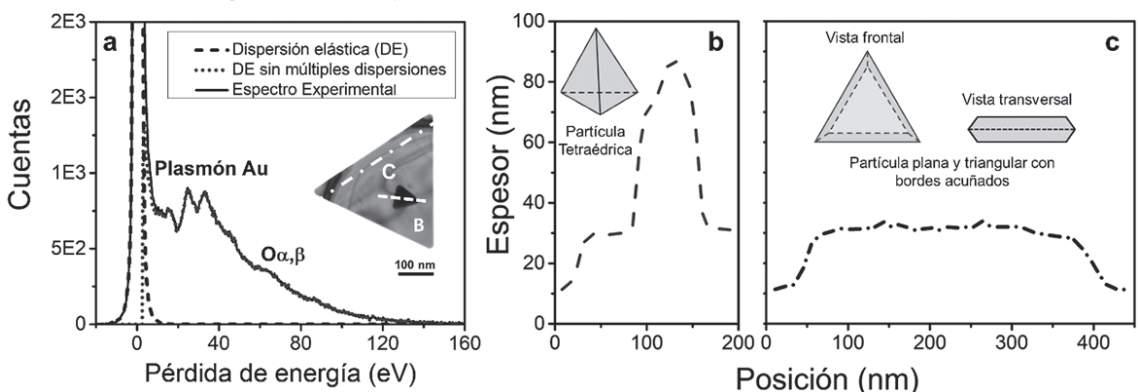

Fuente: Elaboración de los autores. 
para el estudio de la morfología tridimensional de partículas que, analizadas a simple vista, lucen de forma similar.

En la figura 2, se observa otro ejemplo de caracterización típico para el estudio de nanopartículas en campo claro (BF). En la micrografía mostrada en (a) se observa la síntesis de nanobastones en una micrografía BF TEM a bajas magnificaciones. Este tipo de imagen es útil para realizar conteos estadísticos de partículas, tal y como se muestra en el histograma de frecuencias mostrado en la figura 2c. En el acercamiento mostrado en la micrografía (b) se puede observar un ejemplo de contraste por espesor, la barra mostrada, tiene contraste uniforme, dicha propiedad puede ser relacionada con su espesor que, en consecuencia, debe ser constante. Si el perfil del plato no tuviese estas características observaríamos "líneas de extinción", las cuales son ejemplificadas en la figura 3e y 3f, para partículas decaedrales con perfil tipo acuñado.

Figura 2. En (a) se observa la dispersión en tamaño y forma de la síntesis obtenida para la preparación de nanobastón. En (b) puede observarse la morfología típica de un nanobastón, el contraste uniforme en la micrografía es un indicativo de un espesor uniforme. En el histograma mostrado en (c) se muestra que la longitud típica de las barras es de $40-50 \mathrm{~nm}$ en el $40 \%$ de las partículas medidas; finalmente, se muestra un acercamiento a la partícula (d) en el cual es posible observar una imagen en alta resolución, los planos observados en esta imagen pueden relacionarse con los planos $\{002\}$ de Au con distancia interplanar $\mathrm{d}=0.2039 \mathrm{~nm}$.
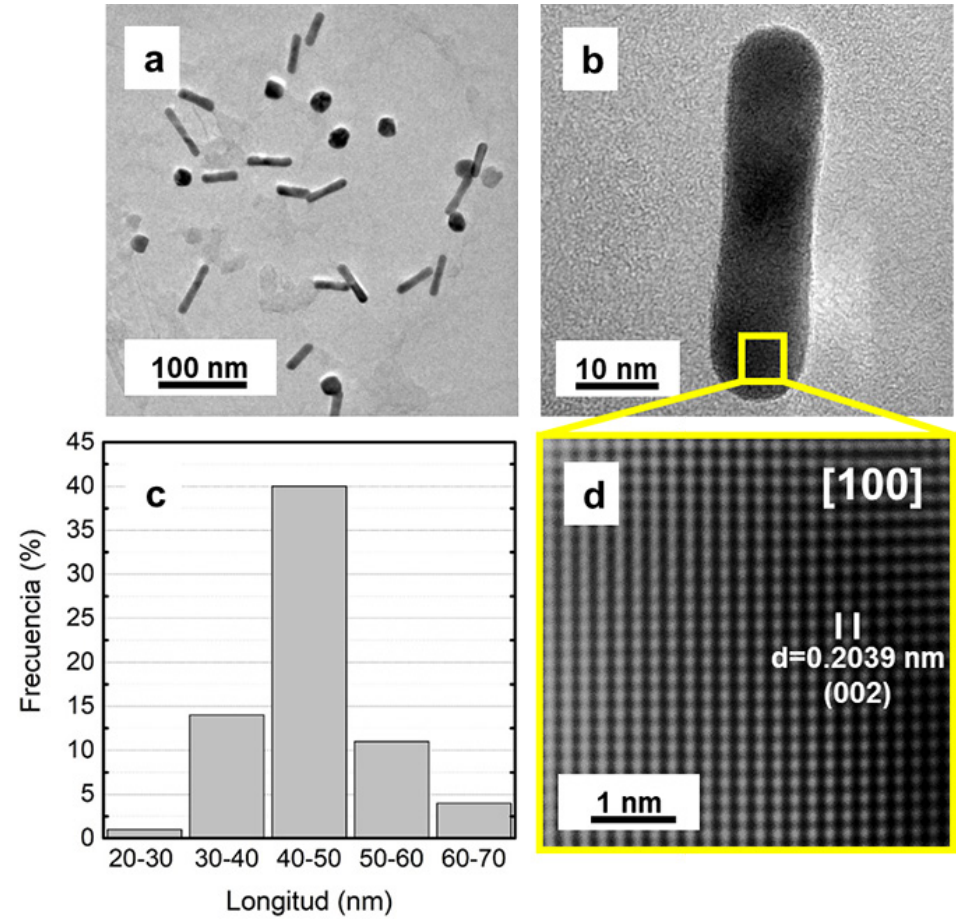

Fuente: Elaboración de los autores. 
Como se comentó en el párrafo anterior, la figura 3 ejemplifica resultados típicos obtenidos para partículas decaedrales, usando dos técnicas distintas: STEM (microscopía electrónica de transmisión en barrido) y TEM $\mathrm{BF} / \mathrm{DF}$.

Las imágenes STEM-HAADF (figura 3 a y b) minimizan el contraste por espesor y difracción, maximizando el contraste por masa, siendo conocida también como técnica de contraste-Z, cuyo nombre hace referencia a la sensibilidad de la técnica ante el número atómico de los elementos analizados. Operacionalmente, esta técnica se caracteriza porque emplea haz convergente, además de sonda muy pequeña conocida como "nanoprobe", la cual barre la muestra para formar una imagen que es detectada por un detector anular. El contraste de este tipo de imágenes se caracteriza por tener un fondo oscuro con muestras contrastadas en tonos de gris, cuya intensidad depende del número atómico. En la figura 3, se pueden apreciar las claras diferencias entre STEM HAADF (a, b) y TEM BF (c-d) para el mismo tipo de partícula. Aunque en el presente escrito únicamente se ilustra la técnica de STEM HAADF, STEM es utilizado ampliamente en otros modos de operación tanto en campo claro como aplicando adquisición tomográfica.

En las imágenes de la figura 3 a y b, puede apreciarse claramente la morfología de las partículas, tanto en vista frontal como en vista transversal, esta forma se muestra también esquemáticamente insertada en las micrografías (a y b). Como puede observarse, las partículas en vista transversal muestran un perfil acuñado, el cual no puede ser inferido directamente en la vista frontal de las imágenes STEM. No obstante, en BF (figura 3e) puede observarse claramente la presencia de "líneas de extinción", estas se muestran paralelas al borde exterior de cada uno de los tetraedros que forman la partícula decaedral, tanto en campo claro como oscuro, este tipo de contraste es característico de muestras con espesor no uniforme, su observación y análisis resulta útil para describir la morfología tridimensional de una partícula, además, otro tipo de defectos, conocidos como maclas (figura $3 d$ ) se aprecian claramente en este tipo de imagen.

En la figura 3, se compara un par de imágenes en BF (d) y DF (f), respectivamente. Ambas técnicas se realizan usando iluminación paralela convencional, como se muestra en el diagrama de rayos de la figura 3, siendo la diferencia principal entre estas técnicas, la alineación de los haces transmitidos o difractados con respecto al eje óptico de microscopio. Pues, para campo claro, se alinea el haz transmitido con el eje óptico del microscopio y se filtran las demás reflexiones mediante el uso de una apertura objetiva, obteniendo imágenes de fondo blanco con contraste en negro.

Mientras que para campo oscuro se alinea el eje óptico del microscopio con alguno de los haces trasmitidos, generando una imagen de fondo negro con contraste en blanco. Una imagen en campo oscuro convencional (figura 3f) puede diferenciarse de una imagen de STEM (que también es campo oscuro) (figura 3a-b), gracias al contraste de difracción el cual se encuentra di- 
Mundo Nano | ARTículos DE INVESTIGACIÓN | www.mundonano.unam.mx

13(25), 61-78, julio-diciembre 2020 | https://doi.org/10.22201/ceiich.24485691e.2020.25.69630

Miriam-Carolina Mendoza Ramírez, Miguel Avalos Borja

Figura 3. En esta figura se analiza la morfología típica de partículas decaedrales usando HAADF (a y b) las partículas mostradas en (a) exhiben el mismo contraste debido a que tienen la misma composición. El contraste mostrado en las imágenes en campo claro (BF) puede relacionarse con la morfología y estructura cristalina. En (e) se observan líneas de espesor características de perfiles acuñados. En campo claro y en campo oscuro pueden observarse claramente la presencia de planos maclados. Los cuales se forman por cinco cristales tetraédricas maclados y presentan el perfil acuñado que se muestra en la figura (b). En (c) se observa una imagen en campo claro en donde pueden observarse varias partículas decaedrales y triangulares. A la derecha se observan diagramas de rayos simplificados que ejemplifican la adquisición de imágenes en campo claro y campo oscuro convencional.
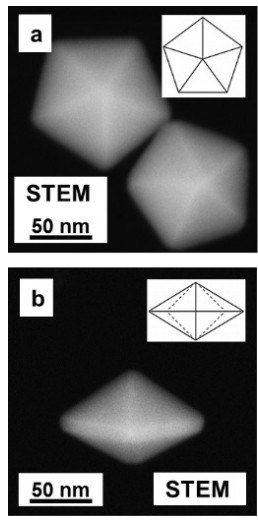
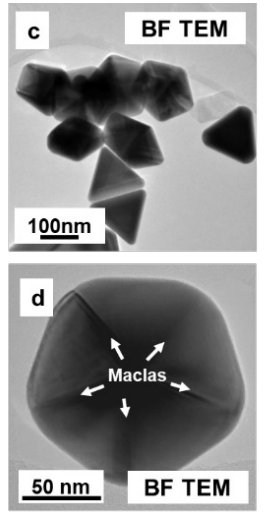

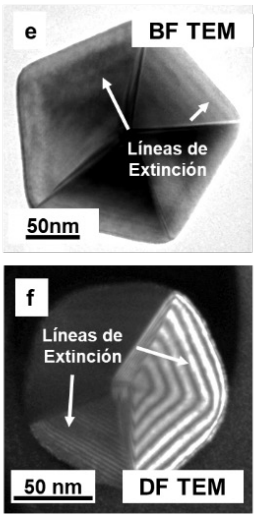

\section{Campo oscuro}

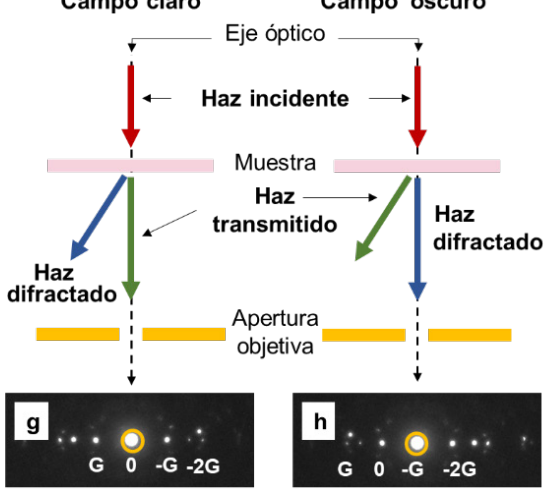

Fuente: Elaboración de los autores.

rectamente relacionado con los haces observados en patrón, lo que permite relacionar las imágenes DF con la estructura de un material.

La relación entre la simetría observada en un patrón de difracción y el contraste en BF puede ser apreciado también de forma clara en el ejemplo mostrado en la figura 4. En esta se muestra un plato de Au de espesor nanométrico (se puede saber que el plato es muy delgado debido a que luce "transparente" al haz de electrones) con morfología hexagonal, este plato delgado tiene una partícula decaedral (mucho más gruesa) que provoca que la partícula se doble, produciendo los contornos de doblado observados en la figura $4 \mathrm{~b}$, los contornos de doblado tienen su origen en la difracción de Bragg, debido a esta, la presencia de estos contornos, en ciertas condiciones de inclinación, mantienen una relación directa con los haces observados en difracción. En la práctica, este tipo de contraste puede ser diferenciado fácilmente pues aparece, desaparece o cambia si se reorienta la muestra.

Además de producir un contraste muy interesante, los contornos de doblado guardan relaciones simétricas que coinciden con los haces observados en difracción. Por ejemplo, en la figura 4b, se seleccionó el cruce de las seis bandas de doblado señalado con la letra (C) usando la apertura de "Área Selecta", obteniendo el patrón de difracción (c) en el cual se observan seis haces difractados con un ángulo de 60 grados entre cada reflexión. De forma similar, al seleccionar el cruce generado por las bandas de doblado indicadas 
con la letra (D) se observa el patrón de difracción (d) en donde se aprecian dos conjuntos de reflexiones principales, separadas a 90 grados. Después de este ejemplo, el lector podrá concluir fácilmente que los contornos de doblado no solo son una linda curiosidad, sino que también resultan muy útiles para orientar muestras en BF y obtener patrones alineados en ejes zonales.

Figura 4. En esta figura se observa un plato delgado y trasparente al haz de electrones con espesor nanométrico. En (b) se muestra un acercamiento de la partícula decaedral b, esta se encuentra sobre el plato delgado y provoca que este se doble, produciendo "contornos de doblado". Los patrones de difracción (c y d) fueron adquiridos en los puntos marcados como C y D, respectivamente en (b).
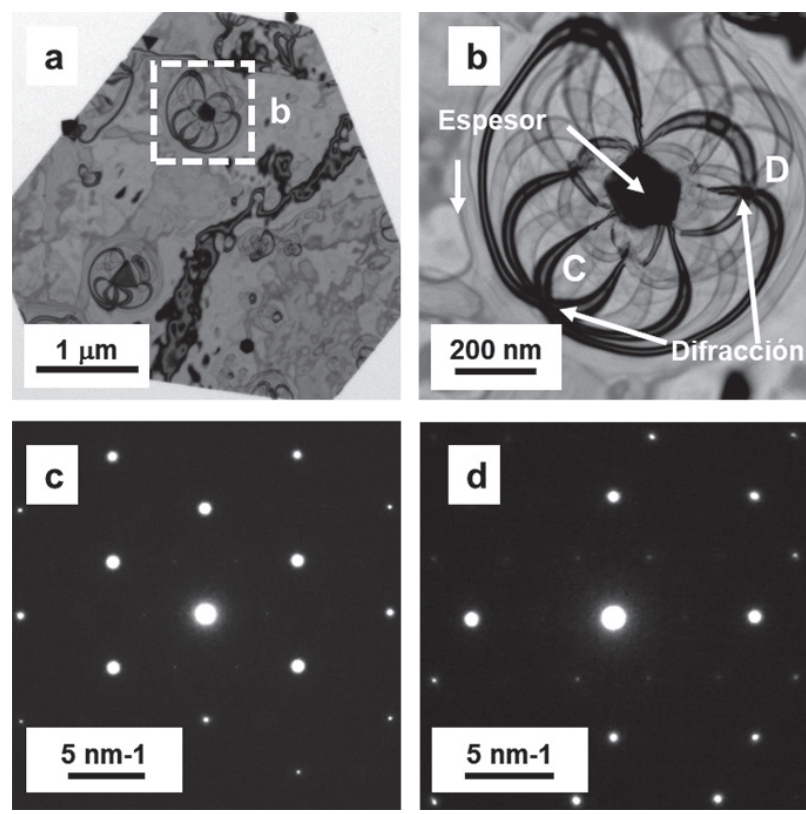

Fuente: Elaboración de los autores.

El contraste por contornos de doblado también puede ser relacionado por sus similitudes con las líneas de Bragg (dinámicas y cinemáticas) presentes en los patrones de difracción de amplio ángulo (LACBED), esta similitud puede ser apreciada en la figuras $7 \mathrm{~d}$ y $8 \mathrm{~d}$, en donde se presentan algunos patrones de difracción adquiridos usando esta técnica.

\section{Difracción de electrones aplicada al estudio de muestras nanométricas}

A continuación, se introducirán de forma breve las diferencias primordiales entre las principales técnicas de difracción empleados en TEM. 
Análisis de patrones SAED (difracción de electrones de área selecta) SAED es la técnica más difundida y una de las más usadas, al permitir obtener calibraciones o identificar el carácter cristalino o amorfo de una muestra. Algunos ejemplos de los patrones típicos que se pueden obtener usando SAED se muestran en la figura 5.

Figura 5. En esta figura se observan distintos tipos de muestras, así como el tipo de patrón de difracción característico que se podría obtener para cada una de estas. (a) Muestra policristalina de calibración y su correspondiente patrón de anillos (e). En (b) se observa la difracción de policristales "no infinitos" como un patrón de anillos discontinuos (f). Cuando se selecciona un solo cristal (c) se puede obtener difracción de monocristal (g). Cuando se observa un material amorfo no se observan puntos intensos, sino que se observa un continuo en espacio recíproco, (la figura (h) es una transformada de Fourier de la imagen (d), equivalente al patrón de difracción).
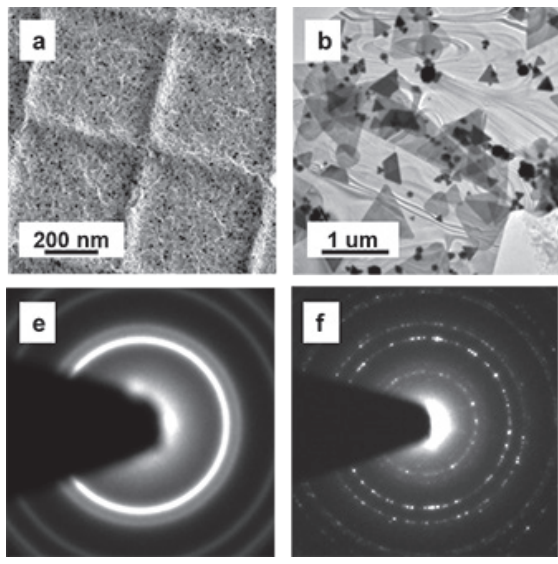
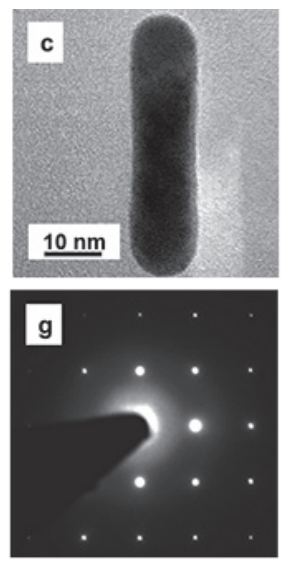
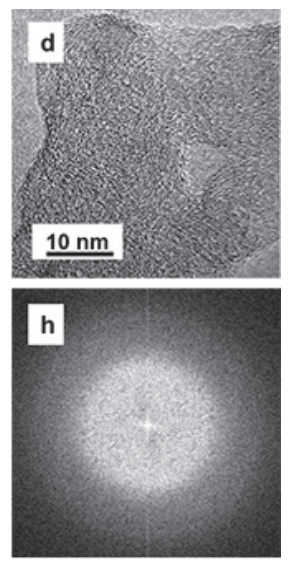

Fuente: Elaboración de los autores.

En la figura 5a, se observa una muestra policristalina cuyo patrón de difracción característico se presenta en la figura 5e, exhibiendo círculos concéntricos en donde cada círculo corresponde a una familia de planos de los cristales en cuestión. Cuando el número de cristales no es "infinito" como en el caso de la figura 5a, el patrón de difracción no muestra anillos continuos sino discontinuos (figuras 5 b y $5 f$ ). Si nos es posible obtener difracción de monocristal, como en el caso de la figura 5c, entonces el patrón de difracción es de una sola "familia" de puntos, como se ve en la figura 5g. Y, por último, si la muestra es amorfa, entonces no hay ni puntos ni anillos, tal como se observa en la figura 5 h.

Para la forma técnica en la obtención de un patrón SAED es preciso iluminar la muestra con un haz de electrones colimado de forma paralela al eje óptico del microscopio (figura 6a). El área a analizar se selecciona por medio de una apertura llamada de "área selecta", la cual se localiza en un plano ópticamente equivalente al plano sobre la muestra, finalmente el patrón se visualiza en la pantalla fluorescente al realizar ajustes en las lentes intermedias. 
A pesar de la belleza de los patrones adquiridos con SAED, su utilidad técnica ha quedado relegada al ser esta técnica muy sensible a efectos dinámicos de "doble difracción", lo cual produce que haya puntos falsos (que no deberían existir) en el patrón de difracción (también llamados "reflexiones prohibidas"). Es por eso que se considera al SAED como una técnica no adecuada para ser relacionada con la estructura cristalina (a través del factor de estructura).

Para ejemplificar este caso, se obtuvo un patrón de difracción SAED en Si (figura 6a); como es sabido, este material tiene grupo espacial 227 con condiciones de reflexión h00: $h=4 \mathrm{n}$. De acuerdo con estas características, la primera reflexión observable en el patrón mostrado en la figura 6a, debería ser la $\{004\}$, sin embargo, se observa que las "reflexiones prohibidas" por grupo espacial $\{002\}$ (que no deberían percibirse) están fuertemente excitadas, debido a efectos dinámicos. Además, puede advertirse que el patrón luce ligeramente desalineado.

Figura 6. En esta figura se ejemplifican (usando diagramas de rayos simplificados) las diferencias en el haz incidente que permiten la obtención de distintos patrones de difracción. (a) Patrón SAED; (b) Patrón PED; (c) CBED, y, (d) Patrones de Kossel.

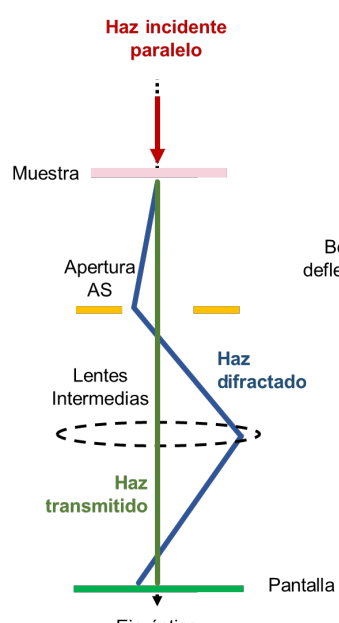

Eje óptico

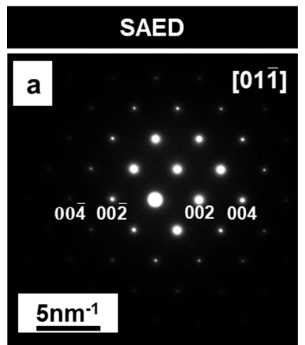

Haz incidente en precesión

(ángulo sólido)
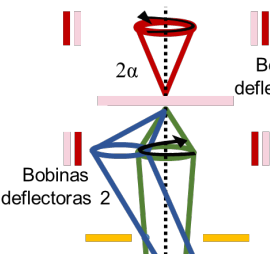

deflectoras 1

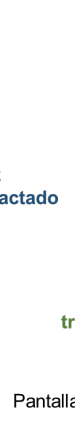

Eje óptico

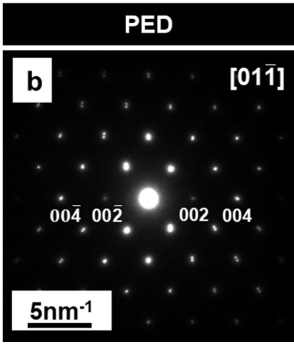

Haz incidente convergente (bajo ángulo)
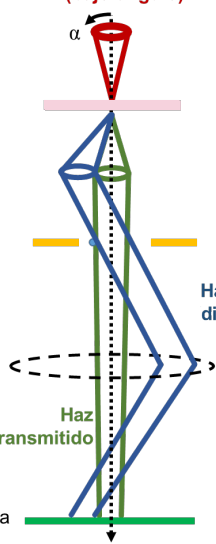

Eje óptico

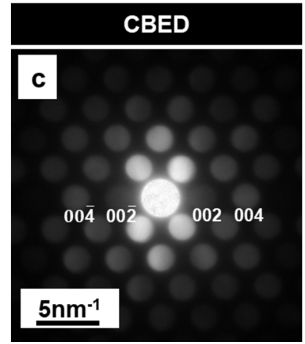

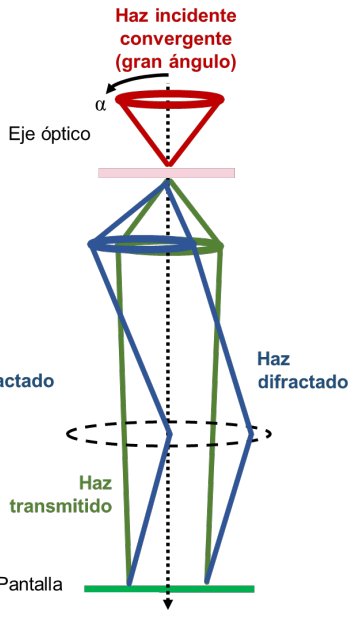

Eje óptico

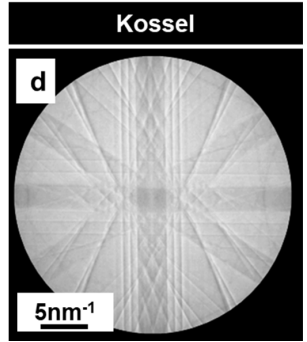

Fuente: Elaboración de los autores. 
Ventajas del uso de PED (difracción de electrones en precesión)

En esta técnica se hace incidir un haz de electrones con cierta inclinación (menor a 5 grados) y de manera cíclica (precesión). Esto se logra modificando las tarjetas electrónicas que controlan la iluminación, a través de dispositivos externos, para nuestro caso se empleó una unidad SpinnigStar P-20. Con este procedimiento se logran minimizar los efectos de difracción dinámica observados en el método SAED. La diferencia principal entre un patrón PED y uno SAED, es que los primeros tienen intensidades adecuadas para ser utilizados en la determinación de estructuras, siendo comparable a la intensidad medida en la difracción de rayos $\mathrm{X}$ convencional. Otra ventaja adicional es que los patrones "lucen" perfectamente alineados, aunque la orientación no sea tan precisa, este detalle puede observarse comparando los patrones a y b de la figura 6 , los cuales fueron adquiridos en las mismas condiciones de orientación. También se observa que en el patrón de difracción PED de la figura $6 \mathrm{~b}$ se disminuye la intensidad de los puntos "prohibidos", cumpliendo con las condiciones de reflexión esperadas para este tipo de cristal.

El uso de técnicas CBED (difracción de electrones con haz convergente) En este caso la iluminación del haz de electrones no es paralela (como en el caso de SAED), sino que es convergente (como se ilustra en la figura 6c). Debido a la convergencia del haz, los "puntos" de difracción no se ven pequeños como en el caso de SAED, sino que lucen como discos. El diámetro de los discos cambia de forma proporcional al ángulo de convergencia de haz. Las técnicas de haz convergente se dividen en técnicas de bajo ángulo, en donde los discos no se sobreponen y de amplio ángulo, en donde los discos difractados crecen tanto que se sobreponen con el haz transmitido.

Usando iluminación convergente también es posible disminuir los efectos dinámicos de la difracción de electrones (similar al modo PED), por lo que las reflexiones prohibidas reducen su intensidad. Los cambios en intensidad ente reflexiones hacen de la técnica CBED una herramienta mucho más útil en comparación a la técnica SAED convencional. Sin embargo, la principal limitación de las técnicas de haz convergente es que se precisan muestras lo suficientemente grandes y gruesas.

\section{Preparación de muestras por FIB (haz de iones enfocados)}

La preparación de lamelas empleadas para las diversas técnicas con haz convergente se ilustra a continuación. La figura 7 (a-d) muestra el proceso de preparación de muestras delgadas (lamelas) preparadas utilizando iones, en un microscopio especial llamado microscopio de iones enfocados o FIB (por sus siglas en inglés). La figura 7a muestra la superficie de silicio con una protección de platino de manera horizontal. En la parte superior y posterior de la protección se hacen "trincheras" utilizando iones de Ga (figura 7b), finalmente, dicha lamela se corta de la placa de silicio utilizando el mismo cañón de iones. Dicha lamela se monta en una rejilla especial utilizando un nano 
manipulador (figura 7c) y se pule y adelgaza con el cañón de iones de Ga, hasta hacerla transparente a los electrones, como puede verse en la imagen de TEM de la figura 7d.

Figura 7. En esta figura se ejemplifica el proceso de preparación de lamelas. Las micrografías (a-c) fueron obtenidas en SEM; (a) muestra el sustrato de Si con Pt; (b) muestra el corte de la lamela, y, (c) La lamela terminada después del desbaste iónico. El borde de la lamela es tan delgado que se dobla. Este detalle se ve claramente en transmisión, pues esta técnica es sensible al contraste de difracción el cual claramente se aprecia en (d) como contornos de doblado.
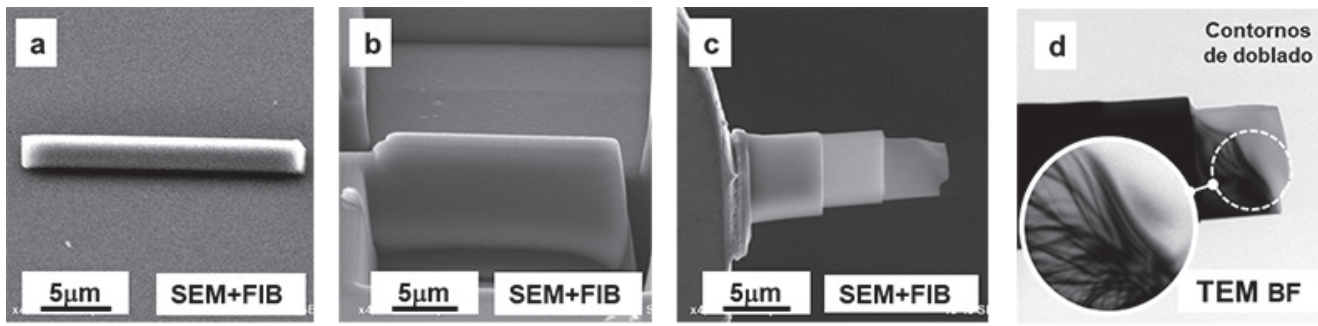

Fuente: Elaboración de los autores.

\section{Difracción LACBED (difracción de electrones con haz convergente de amplio ángulo)}

Usando las técnicas de haz convergente es posible ver información cristalina en cada uno de los círculos observados en el patrón (siempre y cuando la muestra sea lo suficientemente gruesa). Los círculos de difracción observados contienen información referente a la estructura, este fenómeno es conocido como "contraste dinámico" y puede ser observado en el patrón mostrado en la figura 8a. Si un patrón en haz convergente muestra círculos que no se sobreponen se le conoce como patrón de Kossel-Möllenstedt (figura 8a); no obstante, si el ángulo de convergencia de haz aumenta, los círculos comenzarán a sobreponerse, si un patrón llega al punto tal en el que todos los círculos se sobreponen completamente, se conocerá como patrones de Kossel (figuras $6 \mathrm{~d}$ y 8b). En este tipo de patrones se presenta toda la información difractada en un solo disco; aun así, de esta manera es imposible su análisis. Para obtener información útil es preciso, seleccionar únicamente un disco de interés mediante el uso de aperturas, si se selecciona el haz transmitido se obtiene un patrón LACDEB BF (en campo claro) como se muestra en las figuras 8 a y $8 \mathrm{~d}$, por el contrario, si se selecciona alguno de los haces transmitidos se obtiene, un patrón LACBED DF (en campo oscuro).

Debido a que el método de obtención de patrones de amplio ángulo de convergencia (LACBED) es complejo no se discute a profundidad. Sin embargo, sí se resaltan algunas de las maravillas de esta técnica. En la figura $8 c$, se muestra el haz transmitido filtrado para el patrón de Kossel observado en la figura 6d, en este patrón se observa intenso contraste de "líneas de Bragg", las cuales pueden originarse por eventos de difracción dinámica y cinemá- 
Figura 8. En los patrones de difracción (a-d) se muestran varios patrones de difracción con haz convergente. (a) patrón de discos separados (K-M); (b) patrón de discos completamente sobrepuestos o patrón de Kossel; (c) patrón LACBED en campo claro; (d) patrón LACBED, en este último patrón puede observarse de forma simultánea el patrón de difracción y la "sombra de muestra" (lamela mostrada en figura $7 d$ ).

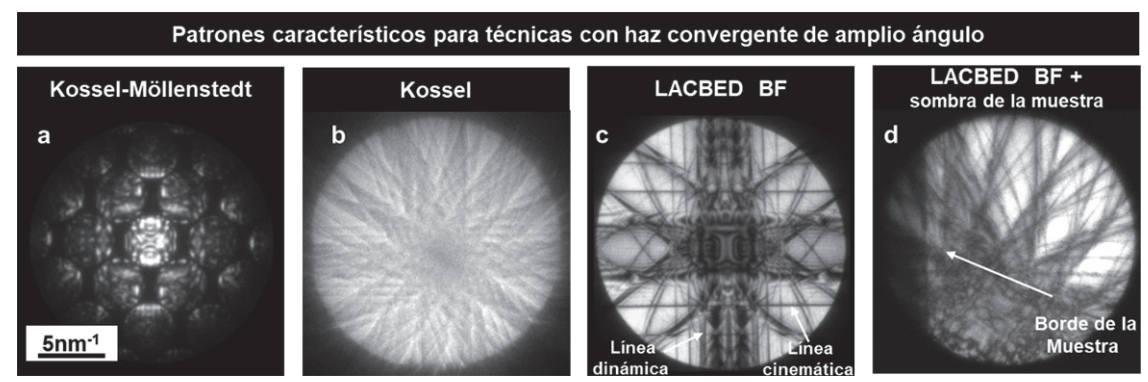

Fuente: Elaboración de los autores.

tica. Estas líneas son particularmente útiles para la caracterización de defectos cristalinos, además, LACBED es la única técnica de difracción que puede mostrar (en ciertas condiciones de foco) la muestra y el patrón de difracción simultáneamente, como se observa en la figura 8d, dicho patrón fue adquirido de la lámela mostrada en la figura 7d, el borde de la lamela puede observarse de manera simultánea al patrón LACEBD en BF.

Tal y como se ilustró a lo largo de este artículo, el microscopio electrónico de transmisión (TEM), provee de un variado grupo de "herramientas" para el estudio de la materia a nivel nanométrico. Con estas herramientas podemos estudiar la morfología de los materiales en 3D (aunque la información de la tercera dimensión la conozcamos de manera indirecta), la química de los materiales (a nivel cualitativo y también cuantitativo), así como la información cristalográfica en cuanto al arreglo de los átomos dentro de las muestras.

\section{Conclusiones}

Se mostró esquemáticamente y mediante el uso de micrografías didácticas la versatilidad del uso de la microscopía electrónica de transmisión para el estudio de nanopartículas y muestras con espesor nanométrico. Se resaltaron los alcances de esta técnica para el estudio de la composición química y la morfología. En la parte estructural se ejemplificaron las diferencias que pueden ser observadas en materiales, policristalinos, monocristalinos y amorfos por difracción de electrones, además de que se mostró como puede identificarse la presencia de ausencias sistemáticas por medio de distintas técnicas de difracción.

Se mostraron esquemáticamente las principales diferencias en la implementación de las técnicas de difracción, SAED, PED, CBED Y LACBED, además, se presentaron ejemplos del contraste característico esperado para cada una de estas técnicas 


\section{Referencias}

Avilov, A. et al. (2007). Precession technique and electron diffractometry as new tools for crystal structure analysis and chemical bonding determination. Ultramicroscopy, 107: 431-444. https://doi.org/10.1016/j.ultramic.2006.09.006

Barmparis, G. D., et al. (2015). I. N. Nanoparticle shapes by using Wulff constructions and first-principles calculations. Beilstein J. Nanotechnol. 6: 361-368.

Daniel, M.-C. et al. (2004). Gold nanoparticles: Assembly, supramolecular chemistry, quantum-size-related properties, and applications toward biology, catalysis, and nanotechnology. Chem. Rev. 104: 293-346. https://doi.org/10.1021/cr030698+

Das, M., et al. (2011). Review on gold nanoparticles and their applications. Toxicol. Environ. Health Sci. 3: 193-205. https://doi.org/10.1007/s13530-011-0109-y

Egerton, R.F. (1986). Electron energy-loss spectroscopy in the electron microscope. Springer Science+Business Media.

Eustis, S. y El-Sayed, M. (2006). Why gold nanoparticles are more precious than pretty gold: noble metal surface plasmon resonance and its enhancement of the radiative and nonradiative properties of nanocrystals of different shapes. Chem. Soc. Rev. 35: 209-217. https://doi.org/10.1039/B514191E

Kan, C. et al. (2010). Synthesis of high-yield gold nanoplates: Fast growth assistant with binary surfactants. J. Nanomater. 2010: 1-9. https://doi.org/10.1155/2010/969030

Major, T. A. et al. (2013). Optical and dynamical properties of chemically synthesized gold nanoplates. J. Phys. Chem. C 117: 1447-1452. https://doi.org/10.1021/ jp311470t

Midgley, P. A. y Eggeman, A. S. (2015). Precession electron diffraction - a topical review. IUCrJ, 2: 126-136. https://doi.org/10.1107/S2052252514022283

Millstone, J. E., et al. (2008). A. Iodide ions control seed-mediated growth of anisotropic gold nanoparticles. Nano Lett., 8: 2526-2529. https://doi.org/10.1021/ nl8016253

Morin, S. A. et al. (2011). Screw dislocation-driven growth of two-dimensional nanoplates. Nano Lett. 11: 4449-4455. https://doi.org/10.1021/nl202689m

Morniroli, J. P. (2003). CBED and LACBED analysis of stacking faults and antiphase boundaries. Mater. Chem. Phys., 81: 209-213.

Morniroli, J. P. (2006). CBED and LACBED characterization of crystal defects. J. Microsc. 223: 240-245. 10.1016/S0254-0584(02)00564-3. https://doi.org/ 10.1111/j.1365-2818.2006.01630.x

Nikoobakht, B. y El-Sayed, M. A. (2003). Preparation and growth mechanism of gold nanorods (NRs) using seed-mediated growth method. Chem. Mater., 15: 19571962. https://doi.org/10.1021/cm0207321

R. Vincent. P. A. Midgley. (1994). Double conical beam-rocking system for measurement of integrated electron diffraction intensities. Ultramicroscopy, 53: 271282. https://doi.org/10.1016/0304-3991(94)90039-6

Sánchez-Iglesias, A. (2006). et al. Synthesis and optical properties of gold nanodeca- 
hedra with size control. Adv. Mater., 18: 2529-2534. https://doi.org/10.1002/ adma.200600475

Tanaka, M., et al. (1980). LACBED. J. Electron Microsc., 29: 408-412.

Tomar, A. et al. (2013). Short review on application of gold nanoparticles. Glob. J. Pharmacol. 7, 34-38. https://doi.org/10.5829/idosi.gjp.2013.7.1.66173

Vigderman, L. et al. (2012). Functional gold nanorods: Synthesis, self-assembly, and sensing applications. Adv. Mater, 24: 4811-4841. https://doi.org/10.1002/ adma.201201690

Yao, Q. et al. (2017). Understanding seed-mediated growth of gold nanoclusters at molecular level. Nat. Commun., 8: 1-10. https://doi.org/10.1038/s41467-01700970-1

Zhang, J., et al. (2005). Surface enhanced Raman scattering effects of silver colloids with different shapes. J. Phys. Chem. B, 109: 12544-12548. https://doi.org/10.1021/ jp050471d

Zhou, S., et al. (2012). Highly active NiCo alloy hexagonal nanoplates with crystal plane selective dehydrogenation and visible-light photocatalysis. J. Mater. Chem., 22: 16858-16864. https://doi.org/10.1039/C2JM32397D

Zou, X., Hovmöller, S. y Oleynikov, P. (2012). Electron crystallography: electron microscopy and electron diffraction. Oxford: Oxford University Press. 